\title{
Noncontingent positive reinforcers retard later escape/avoidance learning in rats
}

\author{
MARK T. WIGHT and RICHARD D. KATZEV \\ Reed College, Portland, Oregon 97202
}

\begin{abstract}
Rats reared in an environment where the occurrence of food, water, and lighting were response independent acquired an escape/avoidance response in a shuttlebox more slowly than rats reared in an environment where these same events were response dependent. The results were discussed in terms of the effects of uncontrollable environmental events on adaptive behavior.
\end{abstract}

Prior exposure to uncontrollable aversive stimuli severely interferes with later instrumental learning under a wide variety of conditions (Maier \& Seligman, 1976). For example, Overmier and Seligman (1967) reported that most dogs exposed to uncontrollable electric shocks in a Pavlovian harness were later unable to learn to escape or avoid shock in a two-way shuttlebox. Seligman and Maier (1967) demonstrated that it was the dog's lack of control over shock, rather than the shock itself, which produced this failure to respond. In exploring the generality of this so-called "helplessness" effect, Rosellini and Seligman (1975) demonstrated that fooddeprived rats which had previously been given uncontrollable shocks had difficulty learning to escape from a "frustrating" situation. Hiroto and Seligman (1975) have also shown that pretreatment with unsolvable cognitive problems interferes with the ability of college students to escape loud tones. These studies indicate that the helplessness effect has considerable generality within an aversive context. The following investigation extends the generality of this effect further by showing that it is not restricted to such situations. Specifically, it demonstrates that prolonged exposure to uncontrollable positive reinforcers also impairs the performance of rats in a situation where it is possible to control aversive stimuli.

\section{METHOD}

\section{Subjects}

Twenty-four experimentally naive rats from the Long-Evans strain were employed in this study. They were the offspring of four randomly selected breeders from the Reed College animal colony.

\section{Procedure}

The basic experimental procedure involved two phases. The first phase was designed to establish different positive reinforcement histories for the rats comprising the two groups. The effect of the different rearing conditions was then tested on adult escape/avoidance responding in a shuttlebox.

Requests for reprints should be sent to Richard D. Katzev, Department of Psychology, Reed College, Portland, Oregon 97202.
Phase 1. To gain precise control over the rat's positive reinforcement history, a technique originally described by Joffee, Rawson, and Mulick (1973) was employed. One group of rats ( $\mathrm{N}=12 ; 8$ males and 4 females) was reared in contingent environments, where pressing separate levers controlled the delivery of all food (one 45-mg Noyes pellet per press), water (approximately $.1 \mathrm{ml}$ per press), and lighting (dim overhead illumination which went on and off with alternate presses). Another group ( $\mathrm{N}=12 ; 5$ males and 7 females) was reared in yoked noncontingent environments which were physically identical to the contingent environments, except that food, water, and lighting were presented independently of the rat's responding and at the same time and frequency as in the contingent group. Consequently, the occurrence of all major reinforcing events for the noncontingent groups was uncontrollable.

At the start of the experiment, one pregnant Long-Evans hooded rat was placed in each of the two contingent and noncontingent environments. These were contained within an apparatus consisting of two pairs of yoked plywood chambers, each of which measured $90.2 \times 60.9 \times 45.7 \mathrm{~cm}$. Each chamber contained three operant manipulanda evenly spaced along one side. Food was delivered by DSI pellet dispensers (PD-109A) and water by dippers manufactured at Reed College. Lighting was provided by a $28-\mathrm{V}$ dc light bulb with a diffuse cover centered in the ceiling of each chamber. An exhaust fan located above the chambers provided ventilation and masking noise $(70 \mathrm{~dB})$ throughout the entire first phase. Within the first 2 days, the pregnant rats in the two contingent chambers independently acquired the necessary barpress responses to provide them with adequate food and water. All four litters were delivered within 5 days. $^{1}$ The litter sizes were reduced, if necessary, to six pups per mother 5 days after each delivery. When the pups reached 30 days of age, the mothers were removed. Fifty days after the respective deliveries, the response requirement for food was increased from FR 1 to FR 5, while the response requirement for water remained at FR 1 throughout the entire first phase. All rats remained undisturbed in their respective environments throughout the entire first phase, except for occasional cleaning which was performed without handling the animals.

Phase 2. When the rats reached 65 days of age, they were given signaled escape/avoidance conditioning trials in a two-way shuttlebox, manufactured by Lehigh Valley Electronics, which measured $43.5 \times 20.0 \times 18.4 \mathrm{~cm}$. The shuttlebox was housed in a sound-attenuating plywood box with a constant level $(60 \mathrm{~dB})$ of white noise present throughout all experimental sessions. Each conditioning trial began with the onset of a tone ( $85 \mathrm{~dB})$ warning signal. Shock $(.4 \mathrm{~mA})$ was scheduled to be delivered $5 \mathrm{sec}$ later. If the rat crossed the center of the shuttlebox in the presence of the warning signal and prior to the onset of shock, the warning signal terminated and shock was avoided. If the rat failed to respond during this time, shock was delivered 


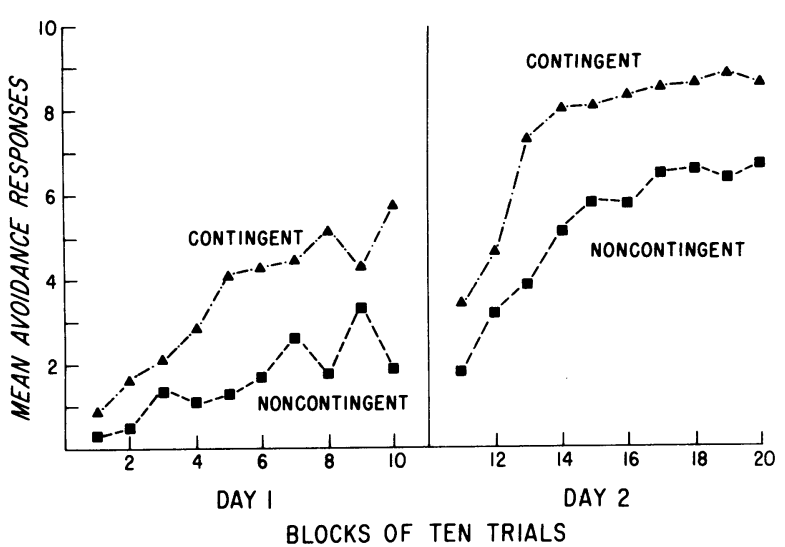

Figure 1. Mean number of avoidance responses for both groups during each block of 10 trials.

and remained on until a response occurred, at which time both the warning signal and shock terminated. If, however, the rat failed to respond within $7 \mathrm{sec}$ after shock onset, the trial was automatically terminated. The trials occurred on a variableinterval schedule with a mean of $30 \mathrm{sec}$ and a range of $10-60 \mathrm{sec}$ All rats received 100 trials of escape/avoidance conditioning per day for 2 consecutive days and were returned to their respective environments at the end of each such session. Stimuli were presented and responses recorded by solid state programming equipment.

\section{RESULTS}

Figure 1 depicts the major outcome of this study in terms of the mean number of avoidance responses during each block of 10 trials for both groups. It is clear that the groups differed in their overall rate of avoidance responding from the very beginning of training. Noncontingent rats acquired the avoidance response more slowly than contingent rats on Day 1 and, while the rate of responding increased for both groups on Day 2, this difference persisted during the second block of 100 trials. Table 1 provides additional performance measures for the rats in the two groups. Separate Mann-Whitney $\mathrm{U}$ tests on these measures revealed that rats reared in the noncontingent environments made significantly fewer avoidance responses on Day $1(U=39, p<.02)$ and Day $2(U=41, p<.04)$ and were significantly slower to escape shock on both Day $1(\mathrm{U}=37.5, \mathrm{p}<.02)$ and Day $2(\mathrm{U}=40.5, \mathrm{p}<.03)$. The noncontingent rats also took significantly longer to reach an arbitrary acquisition criterion of $9 / 10$ avoidance responses $(\mathrm{U}=31, \mathrm{p}<.009)$. In contrast, there were no differences between the groups in their mean weights at the time of testing or in their overall rate of intertrial responding on either Day 1 or Day 2. Finally, the sex of the rat was not significantly related to any performance measures in the shuttlebox.

\section{DISCUSSION}

The findings indicate that control of positive reinforcing events is an important variable which is capable of influencing an organism's ability to escape and avoid shock in the shuttlebox. This evidence also suggests that the degree to which an organism can control early environmental events may play a decisive role in governing their long-term influence on behavior. While the data indicate that the different rearing conditions were sufficient to produce the observed interference in responding, it is impossible to conclude that they were also necessary, because adult rats were not given similar reinforcement histories. Further, while agreeing with Joffee et al. (1973) on the importance of the dimension of control in understanding the effects of early experience, there is no evidence in this study to support their observation that rats reared in noncontingent environments are more "emotional" than rats reared in contingent environments. For example, at no time in this study did the contingent and noncontingent rats differ significantly in terms of their intertrial activity levels in the shuttlebox. Consequently, it is doubtful that the observed differences in this study were a product of this index of emotionality.

It appears that the most parsimonious account of these findings is that recently offered by by Maier and Seligman (1976) for the learned helplessness phenomena. According to this interpretation, learning that reinforcers occurred independently of responding interfered with the ability of rats reared in the noncontingent environments to associate the contingent relationship between their responses and the termination or avoidance of shock in the shuttlebox. If this formulation is valid, the importance of environmental control in regulating subsequent adaptive behavior is not restricted to any single motivational system. Thus, the helplessness effect may be viewed as a general phenomenon which is capable of affecting responding in both appetitive and aversive situations. Consequently, interpretations of helplessness which involve presumed physiological stress reactions (Weiss, Glazer, \& Pohorecky, 1976) are likely to be of limited generality. Indeed, this experiment suggests that uncontrollable positive reinforcers impair responding in an aversive situation in much the same way that uncontrollable aversive events do. Thus, lack of control of both classes of reinforcers may influence a broad range of behaviors and, in so doing, produce a very general impairment in the ability of organisms to learn response-reinforcer relationships.

Table 1

Comparisons Between Contingent and Noncontingent Groups

\begin{tabular}{|c|c|c|c|c|c|c|c|c|c|}
\hline & \multicolumn{2}{|c|}{$\begin{array}{c}\text { Mean Number } \\
\text { Avoidance } \\
\text { Responses }\end{array}$} & \multicolumn{2}{|c|}{$\begin{array}{l}\text { Mean Escape Latency } \\
\text { (in sec) }\end{array}$} & \multirow{2}{*}{$\begin{array}{l}\text { Mean Trials } \\
\text { to Criterion }\end{array}$} & \multicolumn{2}{|c|}{$\begin{array}{c}\text { Mean Number Intertrial } \\
\text { Responses }\end{array}$} & \multicolumn{2}{|c|}{$\begin{array}{l}\text { Mean Weight } \\
\text { (in g) }\end{array}$} \\
\hline & Day 1 & Day 2 & Day 1 & Day 2 & & Day 1 & Day 2 & Males & Females \\
\hline $\begin{array}{l}\text { Contingent } \\
\text { Noncontingent }\end{array}$ & $\begin{array}{l}38.7 \\
17.0\end{array}$ & $\begin{array}{l}72.5 \\
52.6\end{array}$ & $\begin{array}{l}1.26 \\
1.68\end{array}$ & $\begin{array}{r}.88 \\
1.15\end{array}$ & $\begin{array}{r}98.8 \\
152.2\end{array}$ & $\begin{array}{l}64.8 \\
71.2\end{array}$ & $\begin{array}{r}95.1 \\
137.2\end{array}$ & $\begin{array}{l}203.3 \\
208.0\end{array}$ & $\begin{array}{l}158.3 \\
147.4\end{array}$ \\
\hline Mann-Whitney U & $\mathrm{p}<.03$ & $\mathrm{p}<.04$ & $\mathrm{p}<.02$ & $\mathrm{p}<.04$ & $\mathrm{p}<.009$ & n.s. & n.s. & n.s. & n.s. \\
\hline
\end{tabular}




\section{REFERENCES}

Hiroto, D., \& Seligman, M. Generality of learned helplessness in man. Journal of Personality and Social Psychology, 1975, 31, 311-327.

Joffe, J., Rawson, R., \& Mulick, J. Control of their environment reduces emotionality in rats. Science, 1973, 130, 1383-1384.

Maier, S., \& Seligman, M. Learned helplessness: Theory and evidence. Journal of Experimental Psychology: General, 1976, 104, 3-46.

Overmier, J., \& Seligman, M. Effects of inescapable shock upon subsequent escape and avoidance learning. Journal of Comparative and Physiological Psychology, 1967, 63, 28-33.

Rosellini, R., \& Seligman, M. Learned helplessness and escape from frustration. Journal of Experimental Psychology: Animal Behavior Processes, 1975, 1, 149-158.
Seligman, M., \& Maier, S. Failure to escape traumatic shock. Journal of Experimental Psychology, 1967, 74, 1-9.

Weiss, J., Glazer, H., \& Pohorecky, L. Coping behavior and neurochemical changes in rats: An alternative explanation for the original "learned helplessness" experiments. In G. Serban \& A. King (Eds.), Animal models in human psychobiology. New York: Plenum Press, 1976.

\section{NOTE}

1. The pregnant rat in one of the noncontingent chambers cannibalized all its pups 3 days after delivery and was replaced by another pregnant Long-Evans rat which delivered the following day.

(Received for publication December 13, 1976.) 